\title{
Current tobacco use is associated with higher rates of implant revision and deep infection after total hip or knee arthroplasty: a prospective cohort study
}

Jasvinder A. Singh ${ }^{1,2,4^{*}}$, Cathy Schleck ${ }^{4}$, W. Scott Harmsen ${ }^{4}$, Adam K. Jacob ${ }^{3}$, David O. Warner ${ }^{3}$ and David G. Lewallen ${ }^{5}$

\begin{abstract}
Background: Tobacco smoking is a risk factor for several adverse post-operative outcomes. We aimed to compare the rates of complications in current tobacco users and non-users who underwent primary total hip arthroplasty (THA) or total knee arthroplasty (TKA).

Methods: All patients who underwent primary THA or TKA at the Mayo Clinic from 2010-2013 were included in the study. Current tobacco use was defined as the use of cigarettes, cigars, pipes, or smokeless tobacco reported at the time of index THA or TKA; current non-users were former users or never users. We used Cox proportional hazards regression to assess the association of current tobacco use status with each post-THA/TKA complication, using hazard ratios and $95 \%$ confidence intervals (Cl).

Results: Tobacco use status was available for 7926 patients (95\%) and not available for 446 patients (5\%); 565 (7 \%) were current tobacco users. Compared to non-users, current tobacco users were more likely to be male $(p<0.001)$, and less likely to be obese $(p \leq 0.008)$, be older than 60 years, have Charlson score $>0$ or have undergone TKA rather than THA ( $p<0.001$ each). The hazard ratios for deep infection $(2.37 ; 95 \% \mathrm{Cl} 1.19,4.72 ; p=0.01)$ and implant revision $(1.78 ; 95 \% \mathrm{Cl} 1.01,3.13 ; p=0.04)$ were higher in current tobacco users than in non-users. No significant differences were noted for periprosthetic fractures or superficial infections.

Conclusions: We noted that current tobacco use was associated with high risk of deep infection and implant revision after primary THA or TKA. Future studies should determine the optimal time for tobacco use cessation before elective surgeries such as THA and TKA to improve short-term and long-term arthroplasty outcomes.
\end{abstract}

Keywords: Arthroplasty, Complications, Outcomes, Smoker, Smoking, THA, THR, TKA, TKR, Tobacco use, Total hip replacement, Total knee replacement

\section{Background}

Tobacco smoking is a risk factor for several adverse post-operative outcomes, including wound-related complications, in part owing to its effect on tissue oxygenation and inflammatory healing processes $[1,2]$. For

\footnotetext{
* Correspondence: Jasvinder.md@gmail.com

${ }^{1}$ Medicine Service and Center for Surgical Medical Acute Care Research and Transitions, VA Medical Center, Faculty Office Tower, 510, 20th Street South, 805B, Birmingham, AL 35294, USA

${ }^{2}$ Department of Medicine at School of Medicine and Division of Epidemiology at School of Public Health, University of Alabama, 1720

Second Ave. South, Birmingham, AL 35294-0022, USA

Full list of author information is available at the end of the article
}

example, in a study of $>33,000$ patients undergoing total knee arthroplasty (THA) or total hip arthroplasty (TKA) using the Veterans Affairs Surgical Quality Improvement Program database, current smokers had higher risk of 30-day surgical site infections (SSIs) than non-smokers [3]. However, studies describing the association of smoking on short-term or long-term arthroplasty surgical outcomes are limited.

In a study that included 621 patients, the rate of arthroplasty revision was significantly higher in smokers compared to non-smokers in univariate analyses [4]; rates of other surgical complications were no different 
between the groups [4]. However, the analyses were not adjusted for other possibly important covariates such as age, gender, or comorbidity. In contrast, another study that examined the risk of implant revision in 1301 THA patients found no association between smoking and revision risk in ceramic-on-polyethylene bearing primary THA [5]. A systematic review of the effect of smoking on outcomes after total joint replacement showed that current smoking increased the risk of overall postoperative complications and death but that there were scarce data for smoking and surgical outcomes of arthroplasty [6]. To our knowledge, well-designed studies that have examined the risk of post-arthroplasty implant-related complications due to tobacco use are lacking. A recent meta-analysis of studies assessing the effect of smoking on THA outcomes acknowledged the lack of a consistent definition of "current smoker," adjustment for important covariates, and heterogeneity among studies as key limitations [7].

The objective of this study was to assess the association between tobacco use status and outcomes of TKA or THA using data from an institutional Total Joint Registry. We hypothesized that current tobacco use would be associated with an increased risk of revision surgery, wound infections, and peri-prosthetic fractures after primary THA or TKA.

\section{Methods}

We followed the Strengthening of Reporting in Observational studies in Epidemiology (STROBE) statement to describe this study and results [8]. The Institutional Review Board at Mayo Clinic, Rochester, $\mathrm{MN}$, approved the study and waived the requirement for informed consent.

\section{Setting, participants, and data sources}

This observational cohort study included all patients who underwent primary THA or TKA at Mayo Clinic from 2010 to 2013 and had tobacco use status documented in the nursing database. We obtained data from two sources, the Mayo Clinic Joint Registry and the nursing notes of the Mayo Clinic electronic medical record.

The Mayo Clinic Joint Registry is a prospective registry of all patients who undergo joint replacement surgery at the Mayo Clinic, Rochester, MN [9, 10]. Each patient who undergoes joint replacement surgery is followed prospectively with clinical follow-up at one, two, and five years, and every five years thereafter. Patients failing to return for a follow-up visit are sent a questionnaire (joint-specific) and asked to send in their radiographs. Those who fail to return the questionnaire are contacted on the telephone by trained registry staff. Patients undergo a brief telephone interview using a standardized questionnaire including complications, such as infection, fracture, and any additional surgery. Data, including the indication for surgery and operative findings, are requested for subsequent operations performed at other hospitals.

Using unique patient identifiers, we linked the tobacco use status data from the Mayo Clinic Anesthesia database to the Joint Registry. As a matter of routine clinical assessment, tobacco use is consistently documented at the time of admission for patients at all Mayo Clinic hospitals in Rochester, MN, by admission nursing staff. This includes whether patients are currently using tobacco, and the type of use (cigarette, cigar, pipe, or smokeless tobacco use).

\section{Predictor of interest}

Current tobacco use status was the primary predictor of interest. Current tobacco users were defined as people who were using cigarettes, cigars, pipes, or smokeless tobacco at the time of their surgery, with the rest classified as non-users. Thus, current non-users included both never-users and former users.

\section{Covariates}

We included several covariates known/suspected to be associated with complications after THA or TKA, namely demographics (age, gender); body mass index (BMI); American Society of Anesthesiologist (ASA) class; implant fixation (uncemented, cemented, antibiotic/vacuum); and medical comorbidity assessed using a validated Deyo-Charlson index [11], which is a weighted scale of 17 comorbidities (including cardiac, pulmonary, renal, hepatic disease, diabetes, cancer, HIV and so on), expressed as a summative score where a higher score indicates more comorbidity.

\section{Outcomes of interest}

The a priori outcomes were deep infection, superficial infection, peri-prosthetic fracture, and the need for revision surgery. Exploratory outcomes included the reasons for revision (aseptic loosening, infection, and periprosthetic fracture). Deep infection was defined as infection below the fascia; other infections were categorized as superficial infections. Peri-prosthetic fracture was defined as the presence of proximal, distal, or both, or avulsion fracture. Revision surgery was defined as the occurrence of revision of one or more components of the THA or TKA.

\section{Sample size and potential bias}

No formal sample size calculations were done, given the lack of previous studies providing effect size estimates. The Mayo Clinic Joint Registry includes every patient who has undergone THA or TKA at the institution. We anticipated that despite a large sample size, the rarity of these complications would limit the ability to perform 
multivariable-adjusted analyses. We decided a priori that where possible, we would adjust for the most important significant covariates and confounders, and where not possible, acknowledge this as a limitation. We selected a large enough sample by choosing all eligible patients from 2010 to 2013 to avoid a type II error. In very few cases of simultaneous (or sequential) bilateral THA or TKA in a patient, we used only one side (or the first procedure) to avoid correlated observations.

\section{Statistical analyses}

Descriptive statistics were reported as number (percentage) or mean (standard deviation) as appropriate. Characteristics of patients with and without known tobacco use status were compared using logistic regression analyses, also done for current tobacco users versus nonusers. Odds ratios and $95 \%$ confidence intervals (CI) are presented. Cox proportional hazards regression was used to assess the association of tobacco use status with each outcome, reporting a hazard ratio (HR) and $95 \%$ CI. Kaplan-Meier (KM) survival was used to estimate implant survival. The KM curves go to 25 months, because the number at risk drops below 10 patients at 25 months. A $p$-value of less than 0.05 was considered significant.

\section{Results}

\section{Characteristics of patients with and without tobacco use} status data

Tobacco use status was available for 7926 patients (95\%) and not available for 446 patients (5\%) undergoing primary THA or TKA. Compared to those without available tobacco use status assessment, patients with tobacco use data were more likely to be in the age groups $71-80$ and $>80$ years (reference, age $\leq 60$ ), and to be female; no significant differences were noted in BMI, implant fixation, Deyo-Charlson index score, or ASA class (Table 1).

\section{Cohort characteristics}

Of the 7926 patients, 565 (7\%) reported current tobacco use, with 7361 (93\%) not currently using tobacco (including never-users and past tobacco users). In the group of 565 current tobacco users, only two patients were classified as using smokeless tobacco only. In unadjusted analyses, compared to current tobacco nonusers, current tobacco users were more likely to be male $(p<0.001)$, and less likely to be obese $(\mathrm{p} \leq 0.008)$, older than 60 years, have Charlson score $>0$ or have undergone TKA rather than THA ( $\mathrm{p}<0.001$ each). (Table 2$)$. There were no significant differences in implant type or ASA class (Table 2). The mean follow-up was similar between current tobacco user and current tobacco non-users (349 versus 308 days, respectively).

\section{Current tobacco use and the risk of post-THA/TKA complications}

In analyses that adjusted for joint type (THA versus TKA), age, and sex, current tobacco users had significantly higher hazard of deep infection, (HR 2.37; $95 \%$ CI 1.19, 4.72; $p=0.01$ ) and implant revision (HR 1.78; $95 \%$ CI 1.01, 3.13; $p=0.04$ ) than current tobacco nonusers (Table 3). No significant differences in periprosthetic fractures and superficial infection were noted (Table 3). The times free of implant revision and free of deep infection by current tobacco use are shown in Fig. 1a and b, respectively. In exploratory analyses, in a fully adjusted model for joint type (THA versus TKA), age, and sex, current users tended to be at higher risk of revision for infection (HR 2.28; $95 \%$ CI 0.99, 5.27; $p=$ 0.05). Other exploratory outcomes occurred at much lower frequencies that did not allow meaningful analyses to be conducted, that is, revision owing to aseptic loosening occurred only in 14 patients and revision owing to peri-prosthetic fracture in only nine patients.

\section{Discussion}

Smoking can interfere with wound healing and increase infection risk by multiple potential mechanisms, including vasoconstriction, which can interfere with wound healing [12]; fibroblast survival and migration at the site of healing [13]; and carbon monoxide from smoking can lead to decreased oxygen delivery to tissues $[14,15]$, and oxygen is needed for optimal wound healing [16]. In this study of 7926 primary THA or TKA patients, we found that current tobacco use was associated with a significantly higher risk of implant revision and deep infection. These differences remained significant in multivariable-adjusted models that included joint type, age, and gender.

Several prior studies have examined the effect of smoking status on infectious complications in patients undergoing total joint arthroplasty. In a study of 3908 patients with orthopedic implants, including THA and TKA or fracture internal fixation devices, smoking was a risk factor for SSIs for up to one year after surgery [17]. In our previous large study of 33,336 veterans who underwent THA or TKA, current smokers had an adjusted odds ratio of 1.41 for 30-day SSIs compared to never smokers [3]. In the current study, we found that current tobacco users were at 2.37-times higher odds of developing post-surgical deep infection compared to current non-users. Our previous study [3] was performed in veterans, who are sicker than the general US population [18], but the current study cohort is more representative of THA/TKA cohorts. Studies that examined a related, but somewhat differently defined outcome, that is, wound or local complications, reported contradictory results-one study found smoking was associated with wound complications 
Table 1 Comparison of characteristics of patients with and without current tobacco use assessment among patients who underwent primary THA or TKA

\begin{tabular}{|c|c|c|c|c|}
\hline & $\begin{array}{l}\text { Tobacco use assessed } \\
(n=7926)\end{array}$ & $\begin{array}{l}\text { Tobacco use not assessed } \\
(n=446)\end{array}$ & $\begin{array}{l}\text { Odds Ratio } \\
\text { (95\% confidence interval) }\end{array}$ & $p$-value \\
\hline & $N(\%)$ & $\mathrm{N}(\%)$ & & \\
\hline Knee & $4,277(94.5 \%)$ & $249(5.5 \%)$ & $0.93(0.76,1.12)$ & 0.44 \\
\hline Hip & 3,649 (94.9\%) & 197 (5.1 \%) & 1.0 (ref) & \\
\hline Male & $3516(94.1)$ & $219(5.9)$ & $0.82(0.68,0.99)$ & 0.04 \\
\hline Female & $4410(95.1)$ & $227(4.9)$ & 1.0 (ref) $^{a}$ & \\
\hline Age $\leq 60$ & $2430(93.8)$ & $161(6.2)$ & 1.0 (ref) $^{a}$ & \\
\hline $61-70$ & $2522(94.2)$ & $154(5.8)$ & $1.10(0.88,1.39)$ & 0.40 \\
\hline $71-80$ & $2226(95.5)$ & $104(4.5)$ & $1.44(1.12,1.86)$ & 0.005 \\
\hline$>80$ & $748(96.5)$ & $27(3.5)$ & $1.86(1.22,2.81)$ & 0.004 \\
\hline $\mathrm{BMI}<25$ & $1177(95.4)$ & $57(4.6)$ & 1.0 (ref) $^{a}$ & \\
\hline $25-29$ & $2418(94.4)$ & $144(5.6)$ & $0.82(0.60,1.12)$ & 0.21 \\
\hline $30-34$ & $2169(95.2)$ & $109(4.8)$ & $0.97(0.70,1.35)$ & 0.86 \\
\hline $35-39$ & $1235(94.4)$ & $73(5.6)$ & $0.83(0.58,1.18)$ & 0.30 \\
\hline$\geq 40$ & $889(93.8)$ & $59(6.2)$ & $0.74(0.50,1.08)$ & 0.12 \\
\hline Cemented & $2309(94.6)$ & $133(5.4)$ & $1.12(0.71,1.76)$ & 0.62 \\
\hline Antibiotic/vacuum & $2502(94.6)$ & $142(5.4)$ & $1.12(0.74,1.71)$ & 0.59 \\
\hline No cement & $3115(94.8)$ & $171(5.2)$ & $1.0(\text { ref })^{a}$ & \\
\hline Charlson Index $=0$ & $3734(94.6)$ & $212(5.4)$ & $1.0(\text { ref })^{a}$ & \\
\hline$>0$ & $4192(94.7)$ & $234(5.3)$ & $1.02(0.84,1.24)$ & 0.81 \\
\hline$A S A=1$ & $231(95.4)$ & $11(4.6)$ & 1.0 (ref) $^{a}$ & \\
\hline 2 & $5455(94.8)$ & $298(5.2)$ & $0.89(0.48,1.64)$ & 0.70 \\
\hline 3 & $2195(94.3)$ & $133(5.7)$ & $0.80(0.43,1.51)$ & 0.49 \\
\hline 4 & $42(91.3)$ & $4(8.7)$ & $0.51(0.15,1.66)$ & 0.26 \\
\hline
\end{tabular}

ASA American Society of Anesthesiologist, BMl body mass index in $\mathrm{kg} / \mathrm{m}^{2}$, Ref reference category, $S D$ standard deviation

${ }^{a}$ the model was adjusted for the joint type (TKA vs. THA)

with odds of 2.15 [19], while another found no difference in local complications between smokers and non-smokers (3.8 \% versus $3.8 \%$ ) [20]. A systematic review of smoking and outcomes after total joint replacement showed that current smoking increased the risk of any post-operative complication and death [6]. Another systematic review that included any surgery showed that current smoking was associated with SSI and wound complications with odds ratios of 1.79 (95 \% 1.57-2.04) and 2.27 (95\% 1.82-2.84) [2].

Our finding that current smokers were younger and had lower medical comorbidity than current nonsmokers is similar to the younger age and lower ASA class in current smokers undergoing THA or TKA compared to their counterparts in the Veterans Affairs study of $>33,000$ patients [3].

Our study extends these studies of infectious complications by studying all-comers who underwent primary THA or TKA at the Mayo Clinic, using a large sample, examining superficial and deep infections separately, and performing multivariable-adjusted analyses. Even though we had a large sample size, the frequency of some complications was low, which may have led to a type II error, that is, lack of power, potentially explaining some negative findings. An interesting observation was that, even when non-significant, odds ratios for several postoperative complications ranged from 1.5 to 2.0 in current tobacco users.

The most novel finding in our study was that current tobacco users had 1.8-fold higher hazard of the risk of revision arthroplasty than non-users, with 117 cases of implant revision in a cohort of 7926 patients. Our finding in univariate association was confirmed in multivariable-adjusted analyses, suggesting that the finding was robust. Our finding is in contrast to few previous studies [5, 21, 22] and agrees with one recent study [4] showing the association of smoking and revision risk. Espehaug et al. studied 1628 THA patients in a matched case-control study from the Norwegian Arthroplasty Register and found that current smokers had similar 
Table 2 Cohort characteristics of THA/TKA patients with and without current tobacco use

\begin{tabular}{|c|c|c|c|c|}
\hline & Current tobacco user & $\begin{array}{l}\text { Past tobacco user or } \\
\text { never used tobacco }\end{array}$ & Odds ratio & $p$-value \\
\hline & $(n=565)$ & $(n=7361)$ & (95\% confidence interval) & \\
\hline & N (\%) & N (\%) & & \\
\hline Knee & $228(5.0 \%)$ & 4049 (95.0 \%) & $0.55(0.46,0.66)$ & $<0.001$ \\
\hline Hip & 337 (8.8 \%) & 3312 (91.2 \%) & 1.0 (ref) & \\
\hline Male & $326(9.3)$ & $3190(90.7)$ & $1.71(1.44,2.04)$ & $<0.001$ \\
\hline Female & $239(5.4)$ & $4171(94.6)$ & 1.0 (ref) $^{a}$ & \\
\hline Age $\leq 60$ & $354(14.6)$ & $2076(85.4)$ & 1.0 (ref) $^{a}$ & \\
\hline $61-70$ & $143(5.7)$ & $2379(94.3)$ & $0.38(0.30,0.46)$ & $<0.001$ \\
\hline $71-80$ & $56(2.5)$ & $2170(97.5)$ & $0.16(0.12,0.22)$ & $<0.001$ \\
\hline$>80$ & $12(1.6)$ & $736(98.4)$ & $0.10(0.06,0.18)$ & $<0.001$ \\
\hline $\mathrm{BMI}<25$ & $118(10.0)$ & $1059(90.0)$ & 1.0 (ref) $^{a}$ & \\
\hline $25-29$ & $157(6.5)$ & $2261(93.5)$ & $0.66(0.51,0.85)$ & 0.001 \\
\hline $30-34$ & $144(6.6)$ & $2025(93.4)$ & $0.70(0.54,0.91)$ & 0.008 \\
\hline $35-39$ & $90(7.3)$ & $1145(92.7)$ & $0.82(0.61,1.09)$ & 0.17 \\
\hline$\geq 40$ & $51(5.7)$ & $838(94.3)$ & $0.65(0.46,0.92)$ & 0.02 \\
\hline \multicolumn{5}{|l|}{ Implant fixation } \\
\hline Cemented & $124(5.4)$ & 2185 (94.6) & $0.90(0.62,1.29)$ & 0.57 \\
\hline Antibiotic/vacuum & $151(6.0)$ & $2351(94.0)$ & $0.96(0.69,1.32)$ & 0.81 \\
\hline No cement & $290(9.3)$ & $2825(90.7)$ & 1.0 (ref) $^{a}$ & \\
\hline Charlson Index $=0$ & $309(8.3)$ & $3425(91.7)$ & 1.0 (ref) $^{a}$ & \\
\hline$>0$ & $256(6.1)$ & 3936 (93.9) & $0.76(0.63,0.90)$ & 0.001 \\
\hline ASA class 1 & $18(7.8)$ & $213(92.2)$ & 1.0 (ref) $^{a}$ & \\
\hline 2 & $393(7.2)$ & $5062(92.8)$ & $1.05(0.64,1.72)$ & 0.85 \\
\hline 3 & $148(6.7)$ & 2047 (93.3) & $1.00(0.60,1.68)$ & 0.99 \\
\hline 4 & $5(11.9)$ & $37(88.1)$ & $1.72(0.60,4.95)$ & 0.31 \\
\hline
\end{tabular}

ASA American Society of Anesthesiologist, $B M I$ body mass index in $\mathrm{kg} / \mathrm{m}^{2}$, Ref Reference category, SD standard deviation

${ }^{a}$ the model was adjusted for the joint type (TKA vs. THA)

odds of $0.8(95 \%$ CI $0.5,1.3)$ for reoperation as the nonsmokers [21]; study limitations were that smoking status was assessed at the time of survey post-arthroplasty and the definition of "early revision" was unclear. No association between smoking and revision risk was found in
1301 patients (34 revisions) with ceramic-onpolyethylene bearing primary THA, with odds ratio of $1.3(95 \%$ CI 0.6, 2.5) [5]. Meldrum et al. retrospectively studied 147 patients with THA performed by a single surgeon and found that in multivariable models adjusted

Table 3 Adjusted for joint type and multivariable-adjusted hazard of each complication by current tobacco use status

\begin{tabular}{|c|c|c|c|c|c|c|c|c|}
\hline Endpoint & $\begin{array}{l}\text { Current } \\
\text { tobacco user }\end{array}$ & $\begin{array}{l}\text { Number } \\
\text { of events }\end{array}$ & $\begin{array}{l}1 \text { year } \\
(95 \% \mathrm{Cl})\end{array}$ & $\begin{array}{l}2 \text { years } \\
(95 \% \mathrm{Cl})\end{array}$ & $\mathrm{HR}(95 \% \mathrm{Cl})^{\mathrm{a}}$ & $p$-value & $\mathrm{HR}(95 \% \mathrm{Cl})^{\mathrm{b}}$ & $p$-value \\
\hline \multirow[t]{2}{*}{ Any revision } & Yes & 16 & $95.8(93.4,98.3)$ & $93.6(90.5,96.8)$ & $1.98(1.14,3.46)$ & 0.02 & $1.78(1.01,3.13)$ & 0.04 \\
\hline & No & 111 & $98.2(97.8,98.6)$ & $97.3(96.7,97.8)$ & 1.0 (ref) & & 1.0 (ref) & \\
\hline \multirow[t]{2}{*}{ Deep infection } & Yes & 10 & $98.1(96.8,99.4)$ & $97.0(94.9,99.0)$ & $2.45(1.25,4.79)$ & 0.009 & $2.37(1.19,4.72)$ & 0.01 \\
\hline & No & 57 & $98.9(98.6,99.2)$ & $98.7(98.3,99.0)$ & 1.0 (ref) & & 1.0 (ref) & \\
\hline \multirow[t]{2}{*}{ Superficial infection } & Yes & 4 & $99.0(98.1,100)$ & $99.0(98.1,100)$ & $1.06(0.38,2.98)$ & 0.92 & $1.07(0.37,3.09)$ & 0.90 \\
\hline & No & 50 & $99.1(98.8,99.3)$ & $99.1(98.8,99.3)$ & 1.0 (ref) & & 1.0 (ref) & \\
\hline \multirow[t]{2}{*}{ Peri-prosthetic fracture } & Yes & 10 & $98.2(97.0,99.4)$ & $98.2(97.0,99.4)$ & $0.84(0.44,1.58)$ & 0.59 & $1.01(0.53,1.92)$ & 0.97 \\
\hline & No & 134 & $98.0(97.6,98.3)$ & $97.7(97.3,98.2)$ & 1.0 (ref) & & 1.0 (ref) & \\
\hline
\end{tabular}

${ }^{\mathrm{a}}$ Cox models adjusted for knee (versus hip); ${ }^{\mathrm{b}}$ Cox models adjusted for knee (versus hip), male (versus female), and age at surgery (per 1 year). Significant hazard ratios and $p$-values are in bold 

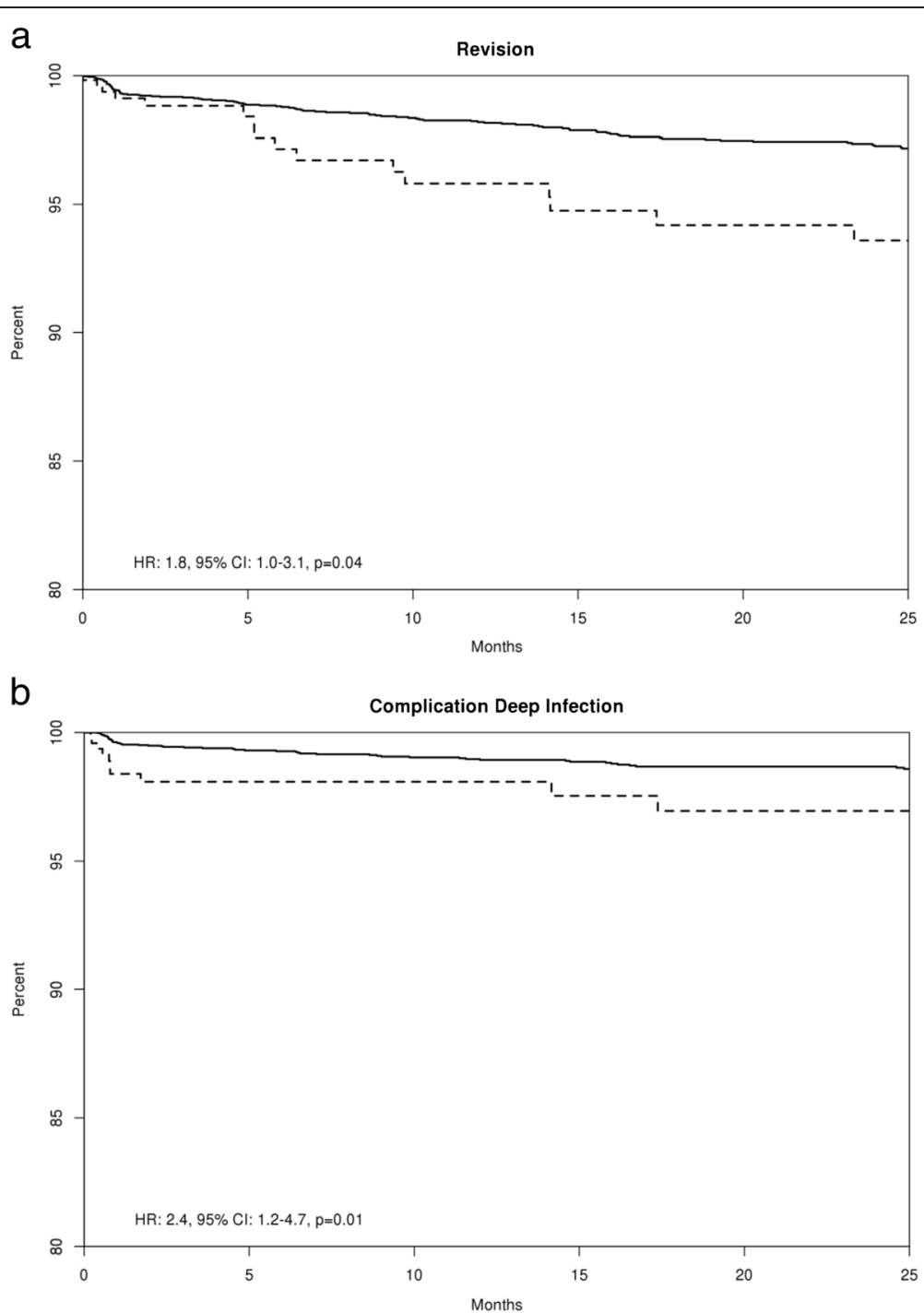

Fig. 1 a Survival free of implant revision for any reason and $\mathbf{b}$ survival free of deep infection in current tobacco users (dashed line) compared with current tobacco non-users (solid line). Cl confidence interval, HR hazard ratio

for age, gender, BMI, diagnosis, stem fixation, and alcohol use, smokers had 4.5-times the hazard of revision than non-smokers, a non-significant finding ( $p=0.07)$ [22]. A single-center study of 621 TKA patients with 18 revisions at mean follow-up of 4 years (131 current smokers with 11 revisions) showed that the risk of revision in smokers was significantly higher than in nonsmokers [4]. Most previous studies of revision risk had small sample sizes leading to potential type II error, that is, missing a significant association when one is present, owing to an underpowered study. Even with $<60$ revisions due to infection, the HR related to current smoking almost reached significance, 2.28 (95\% CI 0.99, 5.27; $p=0.05)$. This correlates well with the biology and the proposed pathophysiology of impaired healing due to tobacco-related toxic effects on fibroblasts and other cells needed for optimal wound healing [12-16].

Our study included a large sample from a Joint Registry, with systematic data collection and monitoring for complications. The higher overall revision risk with current tobacco use may be mediated via a higher rate of associated deep infections in the early post-operative period; the borderline significance of rates of revision for infection further supports this hypothesis.

Our study had several limitations. Findings may not be generalizable to other settings, because this was a singlecenter study. However, the similarity of our THA/TKA cohorts to other published studies of THA/TKA $[23,24]$ as well as the US Nationwide Inpatient Sample [25], 
supports generalizability. Owing to the cohort study design, our study is subject to residual confounding. Confounding due to unmeasured factors among patients undergoing THA/TKA compared to those who are denied the surgery owing to higher medical comorbidity needs to be considered while interpreting study findings. Despite a large sample size, most outcomes of interest were uncommon. Therefore, we likely missed some important associations owing to type II errors, that is, missing an important observation when one existed because of small sample size. Larger sample sizes are needed to definitively answer these questions. Pack-year smoking history was not available for the majority of patients and therefore could not be analyzed.

The number of patients only using smokeless tobacco was extremely small ( 2 of the 565 current smokers), therefore, no subgroup analyses could be performed by the type of tobacco use. As with all observational studies, these findings need to be reproduced in other joint registries of large size (state or country-based registries, Kaiser Permanente etc.) that prospectively assess smoking status and post-arthroplasty complications. Selection bias needs to be considered while interpreting these findings, because patients undergo pre-operative assessment before having THA/TKA and it is possible that those patients who underwent THA/TKA are healthier than the age-matched and sex-matched general population not undergoing these procedures, or patients who undergo less invasive procedures. These findings should not be generalized to the general population or those populations undergoing less invasive procedures.

\section{Conclusion}

We confirmed that current smokers are at increased risk for infectious complications after total joint arthroplasty. In addition, we now show that current smokers are also at risk for adverse functional outcomes that require revision arthroplasty. It is well established that tobacco use interventions reduce perioperative risks in a variety of surgeries (including orthopedic surgery) $[26,27]$. Further studies would be needed to determine if tobacco use intervention specifically can reduce the risk of deep infections and revision surgery. However, because revision is a relatively infrequent event, such studies would need to be large. It would be more practical to have SSI or wound complications as the primary outcome and revision as the secondary outcome. Given the multiple benefits of smoking cessation to both short-term and long-term health, and that most primary TKA and THA are elective, our findings provide further impetus for the routine application of effective tobacco use interventions to all current smokers scheduled for these procedures.

\section{Competing interests}

JAS has received research grants from Takeda and Savient and consultant fees from Savient, Takeda, Regeneron, Merz, Bioiberica, Crealta, and Allergan. JAS serves as the principal investigator for an investigator-initiated study funded by Horizon pharmaceuticals through a grant to DINORA, Inc., a 501 (c)(3) entity. JAS is a member of the executive of OMERACT, an organization that develops outcome measures in rheumatology and receives arms-length funding from 36 companies; a member of the American College of Rheumatology's (ACR) Annual Meeting Planning Committee; Chair of the ACR Meet-the-Professor, Workshop, and Study Group Subcommittee; and a member of the Veterans Affairs Rheumatology Field Advisory Committee. DGL has received royalties/speaker fees from Zimmer, Orthosonic, and Osteotech, has been a paid consultant and owns stock in Pipeline Biomedical, and his institution has received research funds from DePuy, Stryker, Biomet, and Zimmer. Other authors declare that they have no competing interests.

\section{Authors' contributions}

JAS conceived the idea and designed the study, developed study protocol methods and analyses, reviewed statistical analyses, wrote the first draft of the manuscript and performed critical revisions. CS obtained the data, performed data programming and data analysis and revised the manuscript draft. WSH supervised CS in data analysis and reviewed and revised the manuscript draft. AKJ assisted in obtaining data and reviwed and revised manuscript draft. DGL and DOW reviewed the study protocol and statistical analyses and revised the manuscript draft. All authors agreed to submit the manuscript and approved the final manuscript version.

\section{Acknowledgments}

This study was supported by funds from the Mayo Clinic Orthopedic Surgery Department. JAS is supported by the resources and the use of facilities at the VA Medical Center at Birmingham, AL. The funding sources (Mayo Clinic Orthopedic Surgery Department and National Institutes of Health) had no role in study conception, protocol development, data analyses, manuscript preparation, or decision to submit.

\section{Author details}

${ }^{1}$ Medicine Service and Center for Surgical Medical Acute Care Research and Transitions, VA Medical Center, Faculty Office Tower, 510, 20th Street South, 805B, Birmingham, AL 35294, USA. ²Department of Medicine at School of Medicine and Division of Epidemiology at School of Public Health, University of Alabama, 1720 Second Ave. South, Birmingham, AL 35294-0022, USA. ${ }^{3}$ Department of Anesthesiology, Mayo Clinic College of Medicine, 200 1st St SW, Rochester, MN 55905, USA. ${ }^{4}$ Department of Biostatistics, Mayo Clinic College of Medicine, 200 1st St SW, Rochester, MN 55905, USA. ${ }^{5}$ Department of Orthopedic Surgery, Mayo Clinic College of Medicine, 200 1st St SW, Rochester, MN 55905, USA.

Received: 30 August 2015 Accepted: 5 November 2015 Published online: 19 November 2015

\section{References}

1. Sorensen LT. Wound healing and infection in surgery: the pathophysiological impact of smoking, smoking cessation, and nicotine replacement therapy: a systematic review. Ann Surg. 2012;255(6):1069-79.

2. Sorensen LT. Wound healing and infection in surgery. The clinical impact of smoking and smoking cessation: a systematic review and meta-analysis. Arch Surg. 2012;147(4):373-83.

3. Singh JA, Houston TK, Ponce BA, Maddox G, Bishop MJ, Richman J, et al. Smoking as a risk factor for short-term outcomes following primary total hip and total knee replacement in veterans. Arthritis Care Res (Hoboken). 2011;63(10):1365-74.

4. Kapadia BH, Johnson AJ, Naziri Q, Mont MA, Delanois RE, Bonutti PM. Increased revision rates after total knee arthroplasty in patients who smoke. J Arthroplasty. 2012;27(9):1690-5. e1.

5. Lubbeke A, Rothman KJ, Garavaglia G, Barea C, Christofilopoulos P, Stern R, et al. Strong association between smoking and the risk of revision in a cohort study of patients with metal-on-metal total hip arthroplasty. J Orthop Res. 2014;32(6):762-8.

6. Singh JA. Smoking and outcomes after knee and hip arthroplasty: a systematic review. J Rheumatol. 2011;38(9):1824-34. 
7. Teng S, Yi C, Krettek C, Jagodzinski M. Smoking and risk of prosthesisrelated complications after total hip arthroplasty: a meta-analysis of cohort studies. PLoS One. 2015;10(4):e0125294.

8. STROBE Statement. Strengthening the reporting of observational studies in epidemiology. 2007. http://www.strobe-statement.org/fileadmin/Strobe/ uploads/checklists/STROBE_checklist_v4_cohort.pdf. [cited 09/06/2012].

9. Rand JA, Ilstrup DM. Survivorship analysis of total knee arthroplasty. Cumulative rates of survival of 9200 total knee arthroplasties. J Bone Joint Surg Am. 1991;73(3):397-409.

10. Rand JA, Trousdale RT, Ilstrup DM, Harmsen WS. Factors affecting the durability of primary total knee prostheses. J Bone Joint Surg Am. 2003;85-A(2):259-65.

11. Deyo RA, Cherkin DC, Ciol MA. Adapting a clinical comorbidity index for use with ICD-9-CM administrative databases. J Clin Epidemiol. 1992;45(6):613-9.

12. Benowitz NL. Clinical pharmacology of nicotine. Annu Rev Med. 1986;37:21-32.

13. Wong LS, Martins-Green M. Firsthand cigarette smoke alters fibroblast migration and survival: implications for impaired healing. Wound Repair Regen. 2004;12(4):471-84.

14. Heliovaara M, Karvonen MJ, Vilhunen R, Punsar S. Smoking, carbon monoxide, and atherosclerotic diseases. Br Med J. 1978;1(6108):268-70

15. Moucha CS, Clyburn T, Evans RP, Prokuski L. Modifiable risk factors for surgical site infection. J Bone Joint Surg Am. 2011;93(4):398-404.

16. Ninikoski J. Oxygen and wound healing. Clin Plast Surg. 1977;4(3):361-74.

17. Durand F, Berthelot P, Cazorla C, Farizon F, Lucht F. Smoking is a risk factor of organ/space surgical site infection in orthopaedic surgery with implant materials. Int Orthop. 2013;37(4):723-7.

18. Agha Z, Lofgren RP, VanRuiswyk JV, Layde PM. Are patients at Veterans Affairs medical centers sicker? A comparative analysis of health status and medical resource use. Arch Intern Med. 2000;160(21):3252-7.

19. Moller AM, Pedersen T, Villebro N, Munksgaard A. Effect of smoking on early complications after elective orthopaedic surgery. J Bone Joint Surg (Br). 2003;85(2):178-81.

20. Sadr Azodi O, Bellocco R, Eriksson K, Adami J. The impact of tobacco use and body mass index on the length of stay in hospital and the risk of postoperative complications among patients undergoing total hip replacement. J Bone Joint Surg (Br). 2006;88(10):1316-20.

21. Espehaug B, Havelin LI, Engesaeter LB, Langeland N, Vollset SE. Patientrelated risk factors for early revision of total hip replacements. A population register-based case-control study of 674 revised hips. Acta Orthop Scand 1997:68(3):207-15

22. Meldrum RD, Wurtz LD, Feinberg JR, Capello WN. Does smoking affect implant survivorship in total hip arthroplasty? A preliminary retrospective case series. lowa Orthop J. 2005;25:17-24.

23. Huddleston Jl, Wang Y, Uquillas C, Herndon JH, Maloney WJ. Age and obesity are risk factors for adverse events after total hip arthroplasty. Clin Orthop Relat Res. 2012;470(2):490-6.

24. Jimenez-Garcia R, Villanueva-Martinez M, Fernandez-de-Las-Penas C, Hernandez-Barrera V, Rios-Luna A, Garrido PC, et al. Trends in primary total hip arthroplasty in Spain from 2001 to 2008: evaluating changes in demographics, comorbidity, incidence rates, length of stay, costs and mortality. BMC Musculoskelet Disord. 2011;12:43.

25. Kirksey M, Chiu YL, Ma Y, Della Valle AG, Poultsides L, Gerner P, et al. Trends in in-hospital major morbidity and mortality after total joint arthroplasty: United States 1998-2008. Anesth Analg. 2012;115(2):321-7.

26. Thomsen T, Tonnesen $\mathrm{H}$, Moller AM. Effect of preoperative smoking cessation interventions on postoperative complications and smoking cessation. Br J Surg. 2009;96(5):451-61.

27. Tonnesen H, Nielsen PR, Lauritzen JB, Moller AM. Smoking and alcohol intervention before surgery: evidence for best practice. $\mathrm{Br} J$ Anaesth. 2009:102(3):297-306

\section{Submit your next manuscript to BioMed Central and take full advantage of:}

- Convenient online submission

- Thorough peer review

- No space constraints or color figure charges

- Immediate publication on acceptance

- Inclusion in PubMed, CAS, Scopus and Google Scholar

- Research which is freely available for redistribution 